\title{
Wavelets Understand Fractals
}

\author{
Michiel Hazewinkel
}

\begin{abstract}
In this paper I discuss and describe the ideas of analysing fractal structures by means of wavelets as they are presented in $[1-6,8,29,30,35]$ and elsewhere. The wavelet transform can serve as a kind of mathematical microscope to see the fractal nature of various objects at a point and to measure the local scaling symmetry parameters at points.
\end{abstract}

\section{$\S 1$ Fractals (as modeling tools)}

Practically everyone, nowadays, knows what a fractal is; more precisely a fractal set. In one of the more general, rough, definitions it is a set whose Hausdorff-Besicovic dimension is a nonintegral number; whence the name. Examples are the Cantor set of dimension $\frac{\ln 2}{\ln 3}$ and the Koch type curve shown in Figure 1 of dimension 1.5.

Both these examples also show "scaling symmetry": a suitable small part of the whole picture, when magnified, is just like the original. In Figure 2 that is illustrated by the first four generations of the Koch type curve shown in Figure 1. On the left is the so called 'generator'; the first generation is the boundary of the square (second from the left); succeeding generations are obtained from the previous ones by replacing each straight line segment with the generator appropriately scaled down. In Figure 2 the first four generations thus obtained are shown. The limit curve as this process is repeated indefinitely is the fractal set itself; the drawings are of course only crude approximations.

In the following we shall use the word fractal in the sense of something - a set, a function, a measure, ...- that exhibits local scaling symmetry. This is both narrower and wider than the rough first indication definition above: narrower in the sense that scaling symmetry is required; wider in the sense that a figure with scaling symmetry may well have integral dimension. 


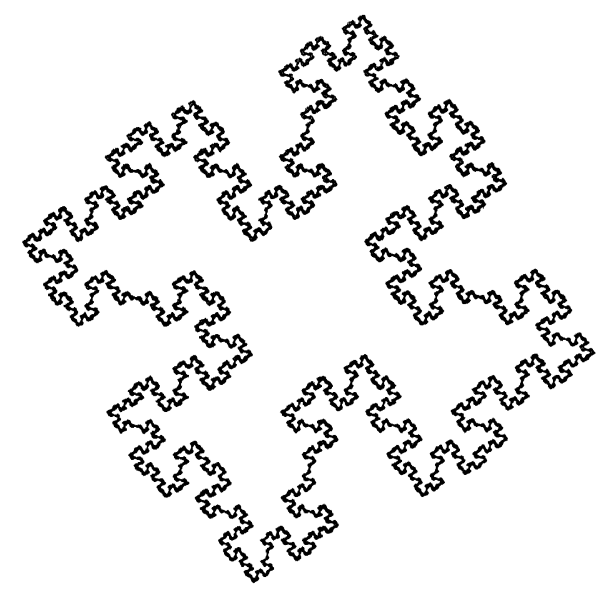

Figure 1. A Koch type curve.

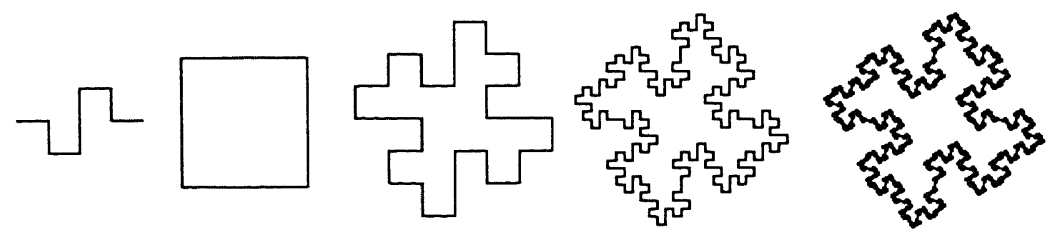

Figure 2. The first four generations of the Koch type curve.

Thus one says that a function $f(x)$ has local scaling symmetry with local scaling exponent $\alpha$ at a point $x_{0}$ if (in a suitable sense)

$$
f\left(x_{0}+a x\right) \approx a^{\alpha} f\left(x_{0}+x\right) \quad \text { as } a \rightarrow 0 .
$$

A measure $\mu$ is said to have local scaling symmetry at $x_{0}$ with exponent $\alpha$ if

$$
\mu\left(I\left(x_{0}, \varepsilon\right)\right)=\int_{I\left(x_{0}, \varepsilon\right)} d \mu(y) \approx \varepsilon^{\alpha}
$$

where $I\left(x_{0}, \varepsilon\right)$ is the interval of length $\varepsilon$ centered at $x_{0}$. Note that if a function with local scaling symmetry at $x_{0}$ is used to define a measure, $d \mu(y)=f(y) d y$, then the scaling exponent shifts by one. As we shall see below the local scaling exponent of a measure is like a dimension.

Here is an example: a so-called two-scale Cantor set (see Figure 3): One starts with the unit interval and uses as a generator the disjoint union of the two subintervals $\left[0, l_{1}\right]$ and $\left[1-l_{2}, 1\right]$, which are given relative weights $p_{1}$ and $p_{2}\left(\right.$ with $\left.p_{1}+p_{2}=1\right)$. 


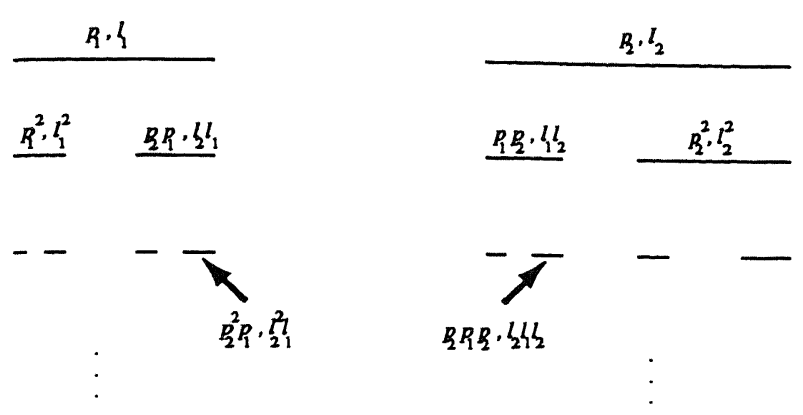

Figure 3. A two-scale Cantor set.

In the illustration above $l_{1}=\frac{1}{4}$ and $l_{2}=\frac{2}{5}$. If one takes $l_{1}=l_{2}=\frac{1}{3}$ and $p_{1}=p_{2}=\frac{1}{2}$ the standard Cantor measure is obtained.

Fractals (fractal sets, fractal measures, fractal functions, ...) have their use as building blocks to model various phenomena. For instance they are used in computer graphics to apply "textures" to graphics models; they are used in image compression schemes, etc.. The most important characteristics of these fractal objects are their local scaling exponents and local dimensions.

Thus it becomes important to take a natural fractal and try to measure these local characteristics. It is here that wavelets can play an important role; they can function as a kind of mathematical microscope to scrutinize the local structure of a fractal. This should not come as a surprise: wavelets already have a well deserved reputation as detectors of singularities or patterns (such as edges in images, or the characteristic sound patterns of submarines (their sound signatures)) also when these are displaced and/or scaled, and thus wavelets should really be particularly good at picking up scaling symmetry structures. And so they are as we shall see below.

\section{$\S 2$ Wavelet transform of a multifractal measure (or function)}

Define a (slightly generalized) wavelet transform by means of the analyzing wavelet $g(x)$ of a measure $\mu$ by the formula

$$
\Phi_{g} \mu(a, b)=M_{g}(a, b)=a^{-n} \int g\left(\frac{x-b}{a}\right) d \mu(x) .
$$

Here the exponent $n$ is chosen so as to best reveal the scaling structure of the measure under consideration. Now suppose that the measure exhibits a scaling symmetry of exponent $\alpha\left(x_{0}\right)$ at the point $x_{0}$ :

$$
\mu\left(I\left(x_{0}, \lambda \varepsilon\right)\right) \approx \lambda^{\alpha\left(x_{0}\right)} \mu\left(I\left(x_{0}, \varepsilon\right)\right) .
$$


Then one has [1-6]:

$$
\begin{aligned}
M_{g}\left(\lambda a, x_{0}+\lambda b\right) & =(\lambda a)^{-n} \int g\left(\frac{x-x_{0}-\lambda b}{\lambda a}\right) d \mu(x) \\
& =(\lambda a)^{-n} \int g\left(\frac{x-\lambda b}{\lambda a}\right) d \mu\left(x+x_{0}\right) \\
& =(\lambda a)^{-n} \int g\left(\frac{x-b}{a}\right) d \mu\left(\lambda x+x_{0}\right) \\
& \approx(\lambda a)^{-n} \int g\left(\frac{x-b}{a}\right) \lambda^{\alpha\left(x_{0}\right)} d \mu\left(x+x_{0}\right) \\
& =(\lambda a)^{-n} \int g\left(\frac{x-x_{0}-b}{a}\right) \lambda^{\alpha\left(x_{0}\right)} d \mu(x) \\
& =\lambda^{\alpha\left(x_{0}\right)-n} M_{g}\left(a, x_{0}+b\right) .
\end{aligned}
$$

Thus a singularity of the fractal measure $\mu$ will manifest itself in terms of a cone like structure in the wavelet transform pointing to the location where the measure has that particular singularity; moreover the scaling exponent can be measured by the corresponding power law divergence in the wavelet transform.

The local nature of the wavelet transform is very essential here. Even so the derivation above is not particularly rigorous; but there are, fortunately, also rigorous results; (see [20-23]).

This is the essential idea in the wavelet analysis of fractal structures.

\section{$\S 3$ Various fractal dimensions}

Let $\alpha(x)$ be the local scaling exponent of the measure $\mu$ at the point $x$; it is also called the 'crowding index'. Let the number of balls of size $\varepsilon$ that have crowding index $\alpha$ be proportional to

$$
\varepsilon^{-f(\alpha)}
$$

as $\varepsilon \rightarrow 0$. It has been shown that $f(\alpha)$ is the Hausdorff-Besicovitch dimension of the subset of points that have local scaling exponent $\alpha[26,39]$. The $f(\alpha)$ are closely related to the Renyi information dimensions $D_{q}$, [24]. These are defined as follows:

$$
D_{q}=\lim _{\varepsilon \rightarrow 0}\left[\frac{1}{q-1} \frac{\ln \mathcal{X}(q)}{\ln \varepsilon}\right], \quad \mathcal{X}(q)=\sum_{i} p_{i}^{q},
$$

where $p_{i}$ is the measure of the $i$-th box when the whole set is chopped up into boxes of size $\varepsilon$. The $f(\alpha)$ and $D_{q}$ are related by a Legendre transform, [26]

$$
\begin{aligned}
& f(\alpha)=\alpha q-(q-1) D_{q} \\
& \alpha(q)=\frac{d}{d q}\left[(q-1) D_{q}\right]
\end{aligned}
$$


In order to calculate or estimate the $D_{q}$ the authors of [26] argue as follows. Divide up a containing set of the support of the fractal measure into subsets of sizes $l_{i}$ of measure $p_{i}$ where the $l_{i}$ are constrained to be smaller than $l$. Write down the partition function

$$
\Gamma(q, \tau, l)=\sum_{i} \frac{p_{i}^{q}}{l_{i}^{\tau}}
$$

It can then be argued that as $l \rightarrow 0$ this sum will remain of order unity if and only if

$$
\tau=(q-1) D_{q} .
$$

(For the case $l_{i}=\varepsilon$ this follows immediately from the definition of the $D_{q}$.)

In the case of a set with a well defined recursive structure, such as a multiscale Cantor set, this can be used to calculate the $D_{q}$. Indeed let the multiscale Cantor measure be defined by the lengths $l_{1}, \ldots, l_{m}$ and the probabilities $p_{1}, \ldots, p_{m}$, then the "level 1" partition function is equal to

$$
\Gamma(q, \tau)=\Gamma_{1}(q, \tau)=\sum_{i} \frac{p_{i}^{q}}{l_{i}^{\tau}}
$$

the level 2 partition function is equal to

$$
\Gamma_{2}(q, \tau)=\sum_{i, j} \frac{\left(p_{i} p_{j}\right)^{q}}{\left(l_{i} l_{j}\right)^{\tau}}=\Gamma(q, \tau)^{2}
$$

and more generally

$$
\Gamma_{n}(q, \tau)=\Gamma(q, \tau)^{n}
$$

This sequence of numbers will remain of order unity if and only if

$$
\Gamma(q, \tau)=1
$$

and from this $\tau(q)$ and hence $D_{q}$ can be calculated.

In the case of the standard Cantor measure, $l_{1}=l_{2}=\frac{1}{3} ; p_{1}=p_{2}=\frac{1}{2}$, this gives the equation

$$
2 \frac{2^{-q}}{3^{-\tau}}=1
$$

and hence $D_{q}=\frac{\ln 2}{\ln 3}$ and the same constant value for $\alpha$ and $f$. The Legendre transformation equations linking these quantities can here be checked directly.

In the case of the 2-scale Cantor measure defined by

$$
l_{1}=\frac{1}{4}, \quad l_{2}=\frac{2}{5} ; \quad p_{1}=\frac{3}{5}, \quad p_{2}=\frac{2}{5},
$$

the equation to be solved is

$$
\frac{p_{1}^{q}}{l_{1}^{\tau}}+\frac{p_{2}^{q}}{l_{2}^{\tau}}=1
$$

which can be done numerically to yield something like the graph in Figure 4 for $D_{q}$ as a function of $q$. Here $D_{-\infty}=1$, and $D_{\infty}=\frac{\ln (0.6)}{\ln (0.25)}=0.3684$. 


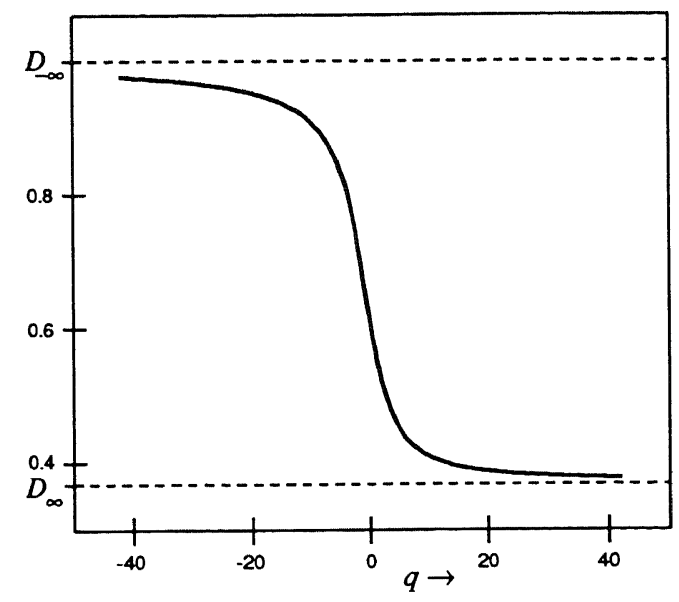

Figure 4. The graph for $D_{q}$ as a function of $q$.

\section{$\S 4$ Wavelet analysis of multiscale Cantor measures}

According to the scaling relation for the wavelet transform $M_{g}(a, b)$ derived in Section 2 above, the strength $\alpha(b)$ of the singularity of the measure $\mu$ at the point $b$ should be reflected in the slope of a $\log -\log$ plot of $\left|M_{g}(a, b)\right|$ versus $a$. Taking $n=2$ in the wavelet transform, this slope should be $\frac{\ln 2}{\ln 3}-2$ for the case of the homogeneous Cantor measure $l_{1}=l_{2}=\frac{1}{3} ; p_{1}=p_{2}=\frac{1}{2}$. And indeed it is, as can be seen from the Figure 5 . More precisely the plot is the wavy continuously drawn line which has the predicted average slope (the dotted line) but oscillates around it with a period of $\ln (2)$. This corresponds to the fact that the scaling relation

$$
\mu\left(I\left(x_{0}, \lambda \varepsilon\right)\right) \approx \lambda^{\alpha\left(x_{0}\right)} \mu\left(I\left(x_{0}, \varepsilon\right)\right)
$$

does not hold for all $\lambda$ but only for a discrete set of values $3^{-n} ; n=1,2, \ldots$. This has been termed lacunarity. (See $[11,12,25,36]$ for discussions of this topic.)

The wavelet transform itself of the homogeneous Cantor measure is depicted in Figure 6 . In this homogeneous case the singularities are everywhere equally strong.

This is no longer the case when we consider an inhomogeneous Cantor measure such as the one defined by $l_{1}=l_{2}=\frac{1}{3}$, and $p_{1}=\frac{3}{4}, p_{2}=\frac{1}{4}$. In this case the strengths of the singularities vary between $a_{\min }=-\frac{\ln p_{1}}{\ln 3}$, the strongest singularity at the left most point, and $a_{\max }=-\frac{\ln p_{2}}{\ln 3}$, the weakest singularity at the right most point. All this follows readily from the same calculations as above for the 2 -scale Cantor measure defined by $l_{1}=\frac{1}{4}, l_{2}=\frac{2}{5}$, and $p_{1}=\frac{3}{5}, p_{2}=\frac{2}{5}$, combined with 


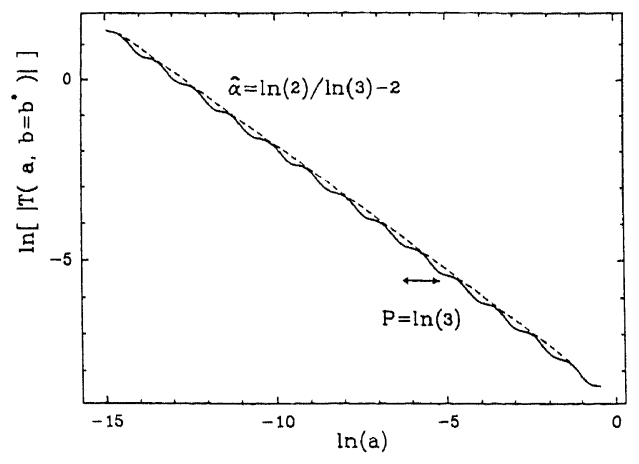

Figure 5

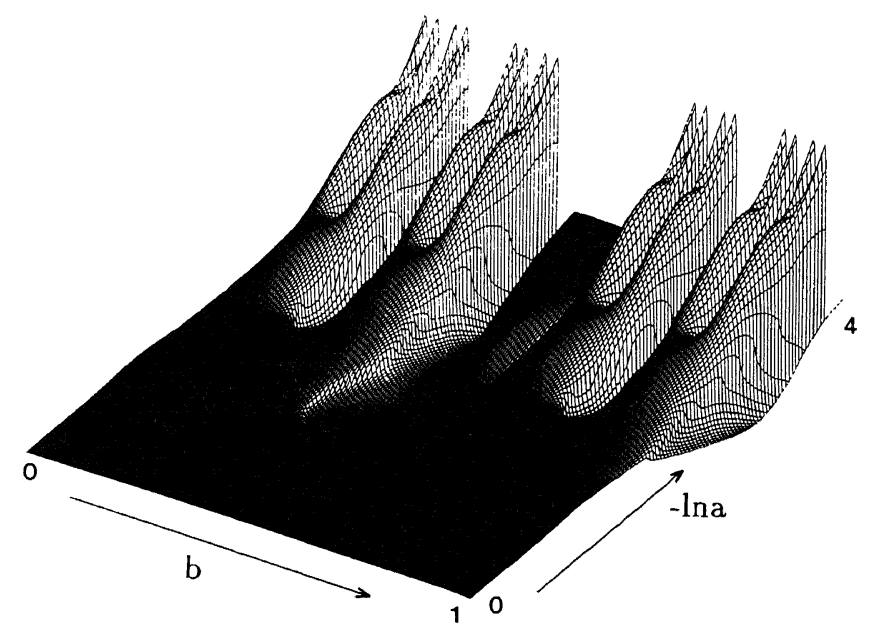

Figure 6

direct calculations or use of the Legendre transform formulas to find the local scaling exponents $\alpha(x)$.

Figure 7 shows the log-log plot of the wavelet transform at the point $b$ that is characterized by the labeling sequence (kneading sequence)

$$
\text { RRRRRRRRLL ..LLL... }
$$




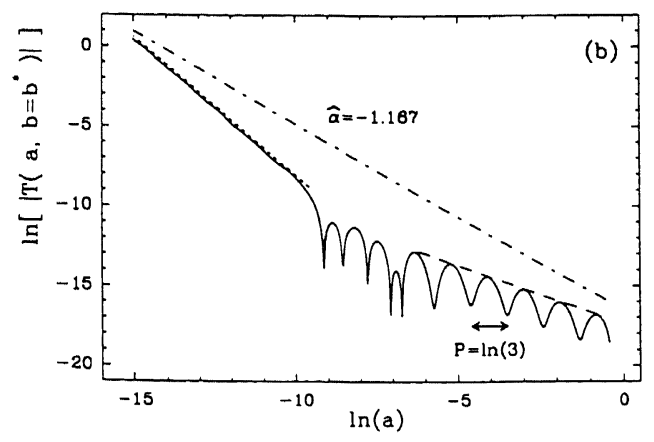

Figure 7

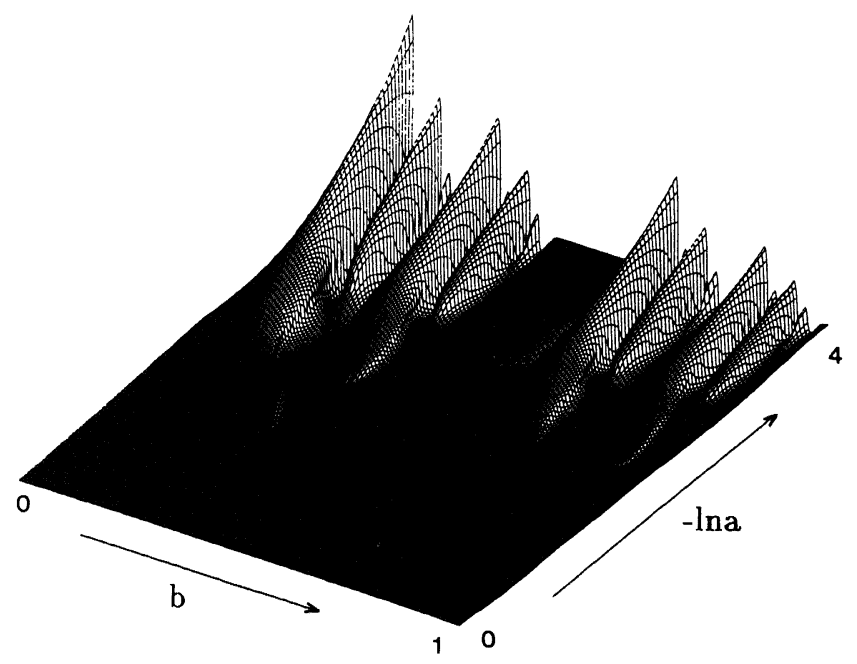

Figure 8

For larger $a$ (on the right in the figure) the average slope is indeed that of the weakest singularity while for small enough $a$ (on the left in the figure) the slope is that of the strongest singularity corresponding to the persistent choice of $L$ in the labeling of the point $b$ after the initial eight $R$ 's.

The period of oscillation in the one but last figure is (of course) $\ln 3$.

The wavelet transform itself of this inhomogeneous Cantor measure is depicted 
in Figure 8. It very clearly shows the differences in the scaling exponents of the measure at the different points of the underlying fractal set.

These last four figures come from (the preprint versions of) $[4,5]$.

\section{$\S 5$ Wavelet analysis of turbulence and various routes to chaos}

Wavelets, more precisely the wavelet transform have also been applied to various routes to chaos such as the period doubling bifurcation cascade, the transition from quasi-periodicity, etc. These analyses, (see e.g. $[5,6]$ for more detail), confirm the fractal nature of these phenomena. Still more interestingly wavelet analysis has been applied to fully developed turbulence (the Richardson cascade) using real life windtunnel data, [3], and the results do point to the fractal nature of fully developed turbulence.

Acknowledgments. Figures 5-8 were reproduced from Figures 3, 4, 6, 7 in [5]. I thank Springer-Verlag for the permission.

\section{References}

1. Argoul, F., A. Arneodo, J. Elezgaray, and G. Grasseau, Wavelet transform of fractal aggregates, Phycics Lett. A 135 (6-7) (1989), 327-336.

2. Argoul, F., A. Arneodo, and G. Grasseau, Fractal dimensions and $f(\alpha)$ spectrum for strange attractors, $Z$ angew. Math. Mech. 68 (10) (1988), 519-522.

3. Argoul, F., A. Arneodo, G. Grasseau, Y. Gagnet, E. J. Hopfinger, and U. Frisch, Wavelet analysis of turbulence reveals the multifractal nature of the Richardson cascade, Nature 338 (1989), 51-53.

4. Arneodo, A., F. Argoul, J. Elezgaray, and G. Grasseau, Wavelet Transform Analysis of Fractals: Application to Nonequilibrium Phase Transitions, Nonlinear dynamics, 1988.

5. Arneodo, A., F. Argoul, and G. Grasseau, Transformation en ondelettes et renormalisation, in Les Ondelettes en 1989, P.G. Lemarié (ed.), LN in Math. 1438, Springer, 1990, 125-191.

6. Arneodo, A., G. Grasseau, and M. Holschneider, Wavelet transforms of multifractals, Physical Rev. Lett. 61 (20) (1988), 2281-2284.

7. Arneodo, A. and M. Holschneider, Crossover effect in the $f(a)$ spectrum for quasiperiodic trajectories at the onset of chaos, Physical Rev. Lett. 58 (20) (1987), 2007-2010.

8. Arneodo, A. and M. Holschneider, Fractal dimensions and homeomorphic conjugacies, J. Stat. Physics 50 (5-6) (1988), 995-1020.

9. Auslander, L. and I. Gertner, Wide-band ambiguity function and $a x+b$ group, in Signal Processing Part I: Signal Processing Theory, L. Auslander, F. A. Grünbaum, J.W. Helton, T. Kailath, P. Khargonekar, S. Mitter (eds.), Springer, 1990, 1-12. 
10. Auslander, L. and R. Tolimieri, On finite Gabor expansions of signals, in Signal Processing Part I: Signal Processing Theory, L. Auslander, F.A. Grünbaum, J.W. Helton, T. Kailath, P. Khargonekar, S. Mitter (eds.), Springer, 1990, 1323.

11. Badii, R. and A. Politi, Intrinsic oscillations in measuring the fractal dimension, Physics Lett. 104A (1984), 303-305.

12. Bessis, D., J.D. Fournier, G. Servizi, G. Turchetti, and S. Vaienti, Mellin transforms of correlation integrals and genralized dimension of strange sets, Physical Review 36A (1987), 920-928.

13. Carrion, P. and K. Bube, One-Dimensional Exact Inversion for Noisy Data, 5-th International Math. Geophysical Sem. 333-340, 1987.

14. Coifman, R.R., Wavelet analysis and signal processing, in Signal Processing Part I: Signal Processing Theory, L. Auslander, F.A. Grünbaum, J.W. Helton, T. Kailath, P. Khargonekar, S. Mitter (eds.), Springer, 1990, 59-68.

15. Collet, P., J.L. Lebowitz, and A. Porzio, The dimension spectrum of some dynamical systems, J. Stat. Physics 47 (5-6) (1987), 609-644.

16. Combes, J.M., A. Grossmann, and Ph. Tchamitchian (eds.), Wavelets. Time Frequency Methods and Phase Space, Springer, 1989.

17. Culik, K. and S. Dube, Methods for generating deterministic fractals and image compression, in Aspects and Prospects of Theoretical Computer Science, LN Comp. Sci. 464, Springer, 1990, 2-28.

18. Doyne Farmer, J., E. Ott, and J.A. Yorke, The dimension of chaotic attractors, Physica D 7 (1983), 153-180.

19. Freysz, E., B. Pouligny, F. Argoul, and A. Arneodo, Optical wavelet transform of fractal aggregates, Phys. Rev. Lett. 64 (1990), 745.

20. Ghez, J-M. and S. Vaienti, On the wavelet analysis for multifractal sets, J. Stat. Physics 57 (1-2) (1989), 415-420.

21. Ghez, J-M. and S. Vaienti, Integrated wavelets on fractal sets. I: the correlation dimension, Nonlinearity 5 (1992), 777-790.

22. Ghez, J-M. and S. Vaienti, Integrated wavelets on fractal sets. II: the generalized dimensions, Nonlinearity 5 (1992), 791-804.

23. Ghez, J-M. and S. Vaienti, Rigorous wavelet analysis for multifractal sets, 1990, Centre de Physique théorique, Marseille, preprint.

24. Grassberger, P. and I. Procaccia, Dimensions and entropies of strange attractors from a fluctuating dynamics approach, Physica $D 13$ (1984), 34-54.

25. Guckenheimer, J., Dimension estimates for attractors. in Fluids and Plasmas: Geometry and Dynamics, J.E. Marsden (ed.), Amer. Math. Soc., 1984, 357367.

26. Halsey, T.C., M.H. Jensen, L.P. Kadanoff, I. Procaccia, and B.I. Shraiman, Fractal measures and their singularities: the characterization of strange sets, Physica. Rev. A 33 (1986), 1141-1151.

27. Heil, C., Wavelets and frames, in Signal Processing Part I: Signal Processing 
Theory, L. Auslander, F.A. Grünbaum, J.W. Helton, T. Kailath, P. Khargonekar, S. Mitter (eds.), Springer, 1990, 147-160.

28. Hentschel, H.G.E. and I. Procaccia, The infinite number of generalized dimensions of fractals and strange attractors, Physica D 8 (1983), 435-444.

29. Holschneider, M., On the wavelet transform of fractal objects, J. Stat. Physics 50 (5-6) (1988), 963-993.

30. Holschneider, $\mathrm{M}$. and $\mathrm{Ph}$. Tchamitchian, Pointwise analysis of Riemanns "nondifferentiable" function, Invent. Math. 105 (1991), 157-175.

31. Lemarié, P.G. (ed.), Les Ondelettes en 1989, Springer, 1990,

32. Lii, K-S and M. Rosenblatt, Nonminimum phase non-gaussian deconvolution, J. Multivariate Anal. 27 (1988), 359-374.

33. Mallat, S.G., A theory for multiresolution signal decomposition: the wavelet representation, IEEE Trans. Pattern Anal. and Machine Intell. 11 (7) (1989), 674-693.

34. Mandelbrot, B.B., Intermittent turbulence in self-similar cascades: divergence of high moments and dimension of the carrier, J. Fluid Mech. 62 (2) (1974), 331-358.

35. Murenzi, R., F. Argoul, A. Arneodo, J. Elezgary, and G. Grasseau, Wavelet transform associated to the $n$-dimensional euclidean group with dilations: application fo the analyis of fractal growth phenomena, 1989, Univ. de Louvain, preprint.

36. Smith, L.A., J-D. Fournier, and E.A. Spiegel, Lacunarity and intermittency in fluid turbulence, Physics Lett. 114A (1986), 465-468.

37. Stark, H-G., Fractal graphs and wavelet series, Physics Lett. A 143 (9) (1990), 443-447.

38. Vicsek, T., Fractal Growth Phenomena, World Scientific, 1989.

39. Vul, E.B., Y.G. Sinai, and K.M. Khanin, Feigenbaum universality and the thermodynamic formalism, Usp. Mat. Nauk 39 (3) (1984), 3-37.

\section{Michiel Hazewinkel}

CWI

P.O. Box 4079

1009 AB Amsterdam

The Netherlands

mich@cwi.nl 